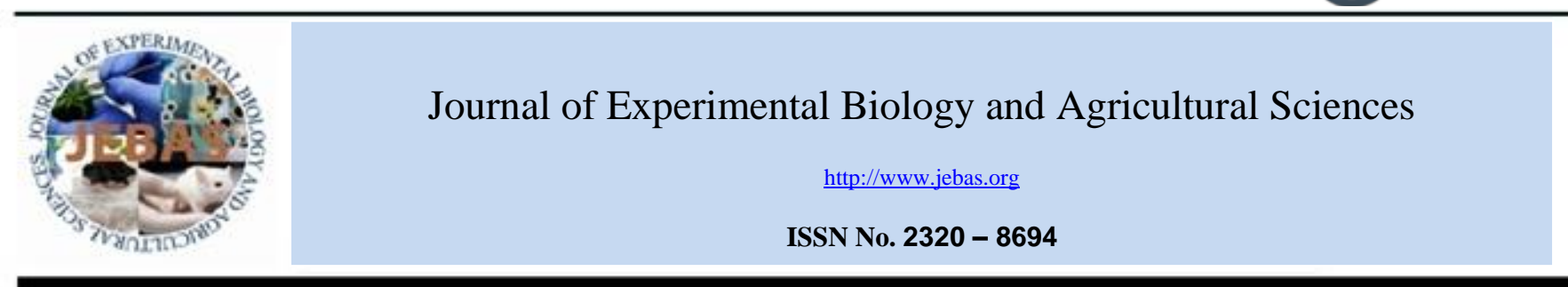

\title{
EFFECTS OF INDIVIDUALIZED TRAINING AND RESPIRATORY MUSCLE TRAINING IN IMPROVING SWIMMING PERFORMANCE AMONG COLLEGIATE SWIMMERS - AN EXPERIMENTAL STUDY
}

\author{
Sivaguru Muthusamy ${ }^{1,2}$, Ambusam Subramaniam $^{1 *}$, K. Balasubramanian ${ }^{2}$ \\ ${ }^{1}$ Physiotherapy programme, INTI International University, Nilai, Negeri Sembilan, Malaysia \\ ${ }^{2}$ Department of Physical Education \& Health Sciences, Alagappa University, Tamil Nadu, India
}

Received - July 18, 2020; Revision - September 17, 2020; Accepted - December 08, 2020

Available Online - March 25, 2021

DOI: http://dx.doi.org/10.18006/2021.9(Spl-1-GCSGD_2020).S125.S132

Keywords

Individualized Training

Respiratory Muscle Training

Collegiate Swimmers

Swimming Performance

\begin{abstract}
Previous researches have been conducted to determine the types of training to improve swimming performance. Nevertheless, no study has been done on the individualized training approach among swimmers. Hence, this study aimed (i) to examine the effects of respiratory muscle training on swimming performance (ii) to examine the effects of combined respiratory muscle training with individualized training on swimming performance, and (iii) to compare the differences between the isolated respiratory muscle training, combined intervention of respiratory muscle training with individualized training and usual training on swimming performance. For this, 45 collegiate swimmers with no previous injuries and swims regularly for at least 1 hour per week participated in the study. Participants were randomly assigned into three groups; Group A: Respiratory muscle training and Individualized Training, Group B: Respiratory muscle training alone, and Group C: Usual training session. The difference within the groups after four weeks of the intervention was analyzed using Paired T-test, while the differences between intervention groups were analyzed using repeated measure twoway ANOVA. Both the intervention groups (Group A and B) showed significant improvement after four weeks of intervention, whereas in group comparison, Group A showed tremendous improvement in swimming performance $\left(\mathrm{F}(17,238)=8.385, \mathrm{p}<0.05, \mathrm{n}_{\mathrm{p}}{ }^{2}=0.375\right)$. Thus, the current study has proven that the combination of respiratory muscle training with individualized training could further enhance the swimming performance in terms of heart rate, Vo2 max, stroke volume, perceived exertion, and SWOLF score. Future studies on athletic swimmers with a larger sample size are recommended to further examine the individualized training approach.
\end{abstract}

* Corresponding author

E-mail:ambusam_7@hotmail.com (Ambusam Subramaniam)

Peer review under responsibility of Journal of Experimental Biology and Agricultural Sciences.

Production and Hosting by Horizon Publisher India [HPI] (http://www.horizonpublisherindia.in/).

All rights reserved.
All the articles published by Journal of Experimental Biology and Agricultural Sciencesare licensed under a Creative Commons Attribution-NonCommercial 4.0 International License Based on a work at www.jebas.org.

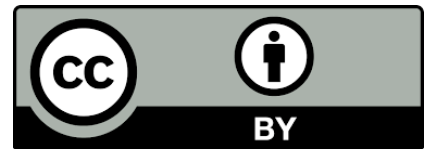




\section{Introduction}

Regular physical activity is the key to maintain health and wellbeing regardless of age. In recent years, people have gained interest in swimming and consider it as a regular form of physical activity. Swimming has been stated to be able to sustain good anthropometric indicators, influence the effect of blood pressure, reduce morbidity risks, and even in general well-being (Cox et al., 2006; Amaro et al., 2017; Cavaggioni et al., 2019). Freestyle swimming is the most common technique which requires proper breathing at various stroke cycle (Tovin, 2006). Swimming, either competitive or amateur, requires good skeletal muscle strength and endurance in addition to strong respiratory muscle function (Lavin et al., 2015). Good respiratory muscle strength and good breathing techniques are needed to complete the whole cycle at a given time. Thus, repetitive activity such as swimming strokes with poor biomechanics and breathing pace may lead to injury and early fatigue, consequently affecting the swimming performance.

Few studies have shown that respiratory muscle training (RMT) able to progress the performance quality among competitive swimmers (Lemaitre et al., 2013; Amaro et al., 2017; Szczepan et al., 2020). Moreover, RMT has the potential benefits in reducing breathlessness, perceived exertion, improving respiratory strength, increase endurance, and enhancing pulmonary function comparing the usual training (Illi et al. 2012; Lemaitre et al. 2013; Yasemin et al., 2019). Similar results were reported in another systematic review with meta-analyses showing that respiratory muscle training can improve sports performance which is highly dependent on training methods employed and the intensity of training (HajGhanbari et al., 2013).

Strength and power are major determinants of swimming performance (Garrido et al., 2010). Individualized training (IT) has the potential to develop respiratory muscle strength, especially under dynamic conditions. Besides, IT can also improve power which represents the ability to perform movements at high speed and/or exert high strength in a short period (Sadowski et al., 2012). The benefits of the IT program were seen via the improvement of the sports performance among various sports fields (Harms, 2007; Illi et al., 2012; HajGhanbari et al., 2013; Jiménez-reyes et al., 2017). Although the effect of the individualized training program is not yet proven among swimmers, limited researches have been carried out on the effects of respiratory muscle training in improving swimming performance (HajGhanbari et al., 2013; Lemaitre et al., 2013; Lavin et al., 2015). Nevertheless, the individualized training performance should also be taken into consideration in improving the swimming performance. However, there is no evidence yet to prove whether individualized training with respiratory muscle training able to further improve swimming performance. Hence, this study aims: (i) to examine the effects of respiratory muscle training on swimming performance among collegiate swimmers, (ii) to examine the effects of combined respiratory muscle training with individualized training on swimming performance among collegiate swimmers, and (iii) to compare the differences between the isolated respiratory muscle training, combined intervention of respiratory muscle training with individualized training, and usual training on swimming performance among collegiate swimmers.

\section{Materials and Methods}

\subsection{Subjects}

Forty-five (23 males and 22 females) collegiate swimmers (Age: $19.58 \pm 1.118$ ) were recruited for the current study. The participants were categorized into three groups i.e. two experimental and one control group using computerized randomization $(n=15$ each group). The participants met all the inclusion and exclusion criteria.

The swimmers were freestyle swimmers, aged between 18-21, and swim regularly for at least 1 hour in total per week. Participants will be excluded from the study if they have any central nervous systems (CNS) disorders such as seizure, epilepsy, multiple sclerosis; respiratory disorders; previous injuries involving upper and lower limbs; BMI greater than $24 \mathrm{~kg} / \mathrm{m}^{2}$; and those who are undergoing other training regimens aside from swimming. All the participants were provided with written informed consent in this study. The research was conducted following the ethics committee of INTI International University.

\subsection{Experimental design}

All the participants were assigned through randomized allocation into three groups; two experimental groups and one control group. The experimental groups consist of Group A: Respiratory muscle training (RMT) and Individualized training (IT), Group B: Respiratory muscle training (RMT), and Group C: Usual training session (TS). The measurements of the swimming performance were taken at two periods viz. (i) Before the intervention period and (ii) After 4 weeks of the intervention of RMT, IT, and TS respective to the groups.

During the first period, a baseline measurement is taken, all the participants were required to swim $100 \mathrm{~m}$ distance. The baseline measurement consists of the time taken to complete $100 \mathrm{~m}$ distance, max HR, VO2 max, strokes, and Swim Golf (SWOLF). The BORG Scale of perceived exertion was recorded at the starting and upon completion of a $100 \mathrm{~m}$ distance of swimming. In the second phase, the participants were randomly allocated into three groups (Group A, B, and C). The participants were given 4 weeks of training based on the group respectively and the measurements were taken again at the end of the 4 th week. All three groups were assessed at the same time. 
Table 1 Baseline characteristics based on the group allocation (Week 1)

\begin{tabular}{|c|c|c|c|}
\hline & Group A $(n=15)$ & Group B $(n=15)$ & Group $C(n=15)$ \\
\hline $\begin{array}{c}\text { Gender }(\mathrm{n} / \%) \\
\text { Male } \\
\text { Female }\end{array}$ & $\begin{array}{l}7(46.7) \\
8(55.3) \\
\end{array}$ & $\begin{array}{l}8(53.3) \\
7(46.7) \\
\end{array}$ & $\begin{array}{l}8(53.3) \\
7(46.7) \\
\end{array}$ \\
\hline Age & $19.6 \pm 1.12$ & $19.53 \pm 1.19$ & $19.6 \pm 1.12$ \\
\hline Time taken for $100 \mathrm{~m}$ distance & $3.66 \pm 0.30$ & $3.56 \pm 0.34$ & $3.49 \pm 0.48$ \\
\hline Max HR & $161 \pm 12.49$ & $165.67 \pm 13.8$ & $168.13 \pm 10.00$ \\
\hline Vo2 max & $41.93 \pm 3.47$ & $42.53 \pm 2.67$ & $41.13 \pm 2.00$ \\
\hline Borg scale & $5.27 \pm 0.70$ & $5.20 \pm 0.68$ & $4.80 \pm 0.94$ \\
\hline Strokes & $79.4 \pm 5.26$ & $79.33 \pm 6.14$ & $76.67 \pm 5.82$ \\
\hline Swim golf (SWOLF) & $298.72 \pm 20.50$ & $292.93 \pm 21.30$ & $286.11 \pm 32.15$ \\
\hline
\end{tabular}

All the data presented in mean \pm standard deviation except gender presented in frequencies (percentage); $\mathrm{HR}=\mathrm{Heart}$ rate; $\mathrm{VO}_{2}$ max $=$ maximal oxygen consumption

Table 2 Measures of swimming performance before and after respiratory muscle training (RMT)

\begin{tabular}{|c|c|c|c|c|}
\hline & $\begin{array}{l}\text { Pre-training } \\
(\text { Mean } \pm \text { SD })\end{array}$ & $\begin{array}{l}\text { Post-training } \\
(\text { Mean } \pm \mathrm{SD})\end{array}$ & Mean differences & $\begin{array}{l}\text { 95\% CI for the Mean (Lower } \\
\text { to Upper) }\end{array}$ \\
\hline Time taken for $100 \mathrm{~m}$ distance & $3.56 \pm 0.34$ & $3.48 \pm 0.35$ & 0.075 & -0.06 to 0.208 \\
\hline Max HR & $166.67 \pm 13.80$ & $156.0 \pm 15.63$ & $9.667 *$ & 6.247 to 13.06 \\
\hline $\mathrm{VO}_{2} \max$ & $42.53 \pm 2.67$ & $46.73 \pm 2.66$ & $-4.200 *$ & -5.527 to -2.873 \\
\hline Borg scale & $5.20 \pm 0.67$ & $4.20 \pm 0.78$ & $1.000 *$ & 0.581 to 1.419 \\
\hline Strokes & $79.33 \pm 6.13$ & $70.07 \pm 4.86$ & $9.267 *$ & 7.117 to 11.416 \\
\hline Swim golf (SWOLF) & $292.93 \pm 21.30$ & $279.15 \pm 21.45$ & $13.787 *$ & 5.94 to 21.63 \\
\hline
\end{tabular}

$* \mathrm{p}<0.05 ; \mathrm{HR}=$ Heart rate; $\mathrm{VO}_{2} \max =$ maximal oxygen consumption

Table 3 Measures of swimming performance before and after combined respiratory training and individualized training (RMT+IT)

\begin{tabular}{|ccccc|}
\hline & $\begin{array}{c}\text { Pre-training } \\
(\text { Mean } \pm \text { SD) }\end{array}$ & $\begin{array}{c}\text { Post-training (Mean } \pm \\
\text { SD) }\end{array}$ & $\begin{array}{c}\text { Mean } \\
\text { differences }\end{array}$ & $\begin{array}{c}95 \% \text { CI for the Mean (Lower to } \\
\text { Upper) }\end{array}$ \\
\hline $\begin{array}{c}\text { Time taken for } 100 \mathrm{~m} \\
\text { distance }\end{array}$ & $3.66 \pm 0.30$ & $3.47 \pm 0.36$ & 0.185 & -0.070 to 0.441 \\
\hline Max HR & $161.00 \pm 12.49$ & $153.73 \pm 12.71$ & $7.267 *$ & -8.538 to -5.462 \\
\hline VO $_{2}$ max & $41.93 \pm 3.47$ & $48.93 \pm 3.86$ & $-7.00^{*}$ & 1.192 to 2.008 \\
\hline Borg scale & $5.27 \pm 0.70$ & $3.67 \pm 0.62$ & $1.600^{*}$ & 8.523 to 12.943 \\
\hline Strokes & $79.4 \pm 5.26$ & $68.67 \pm 4.61$ & $10.733^{*}$ & 6.436 to 37.27 \\
\hline Swim golf (SWOLF) & $298.72 \pm 20.50$ & $276.87 \pm 23.14$ & $21.853^{*}$ & \\
\hline
\end{tabular}

$* \mathrm{p}<0.05 ; \mathrm{HR}=$ Heart rate; $\mathrm{VO}_{2} \max =$ maximal oxygen consumption 


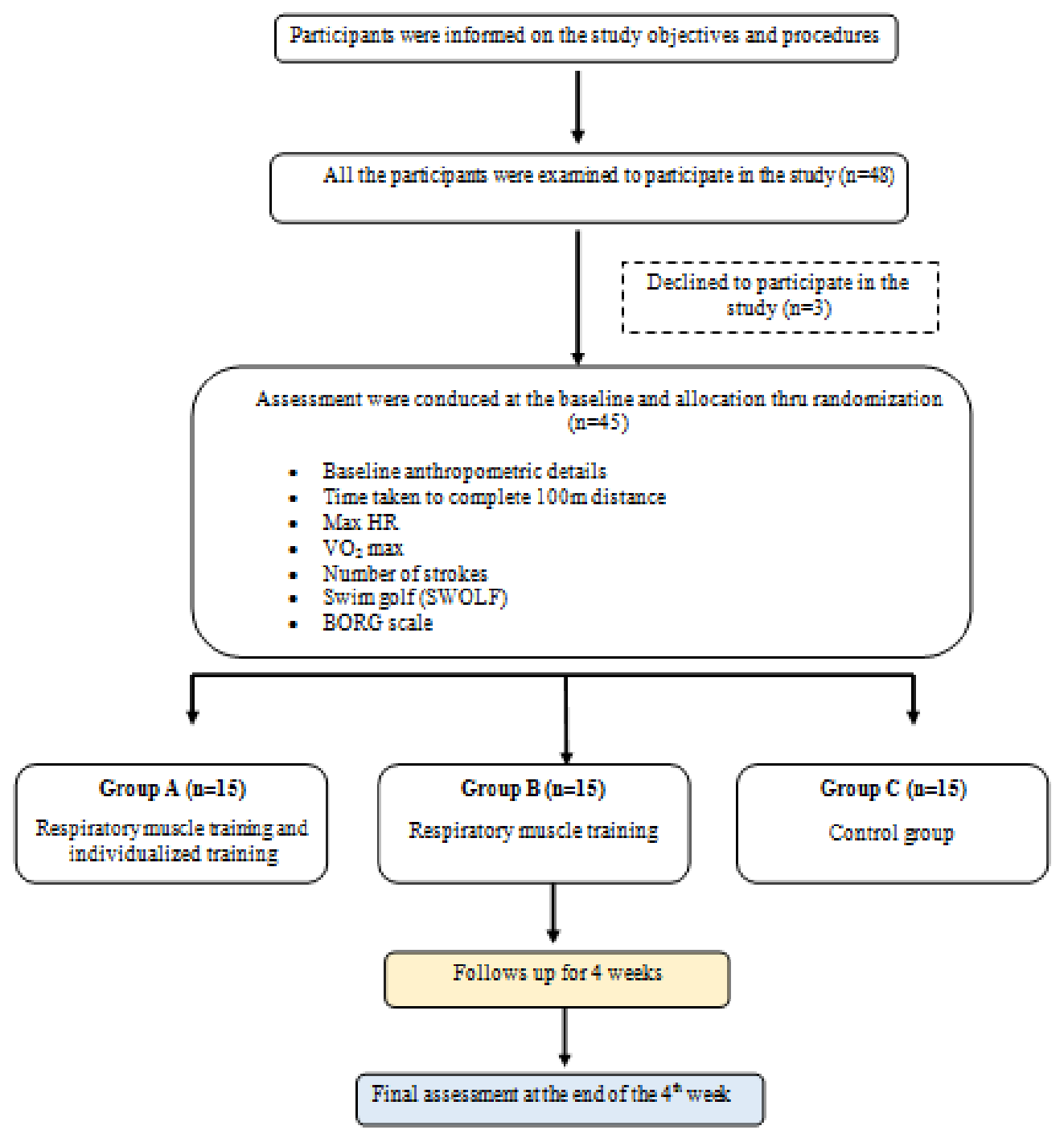

Figure 1 Study Flow chart

\subsection{Training protocol}

Once the participants allocated to the groups, information and procedure regarding the training sessions were informed. For Group A which is RMT+IT, the individualized training intensity based on each participants resting heart rate, maximal heart rate (220-Age), and 10 point Borg's scale was used to prescribe the training intensity (Sadowski et al., 2012; Grant \& Kavaliauskas, 2017). Training intensity was fixed at $70 \%-85 \%$ of maximal heart rate (aerobic zone) (Grant \& Kavaliauskas, 2017). During the first week of training, exercise intensity started with $75 \%$ and gradually progressed based on the individual's capacity. These individualized training also incorporated with RMT throughout the exercise sessions. The intensity for the RMT was prescribed from low level to moderate level (Based on the medium resistance power breathe trainer) (Cunha et al., 2019).

For Group B (RMT) throughout the training session starting from warm-up to cool down, respiratory muscle training was given and participants were instructed to continue their routine day-to-day activities (Lemaitre et al., 2013; Cunha et al., 2019). While for group C participants with routine training sessions were provided with a common exercise load and general land based training protocol prescribed by a swimming coach (Garrido et al., 2010). The core training session includes an aerobic training period such as jogging, cycling (static and non-static), and moderate resistance training focusing on the major muscles of freestyle swimming (Garrido et al., 2010). 


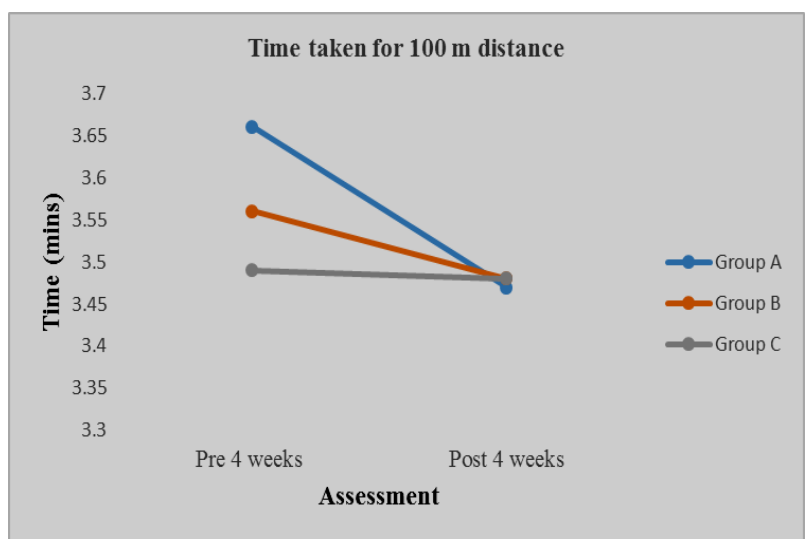

Figure 2 The time taken for subjects to complete $100 \mathrm{~m}$ distance before and after four weeks of intervention, *Pre-post comparison, $\mathrm{P} \leq 0.05$

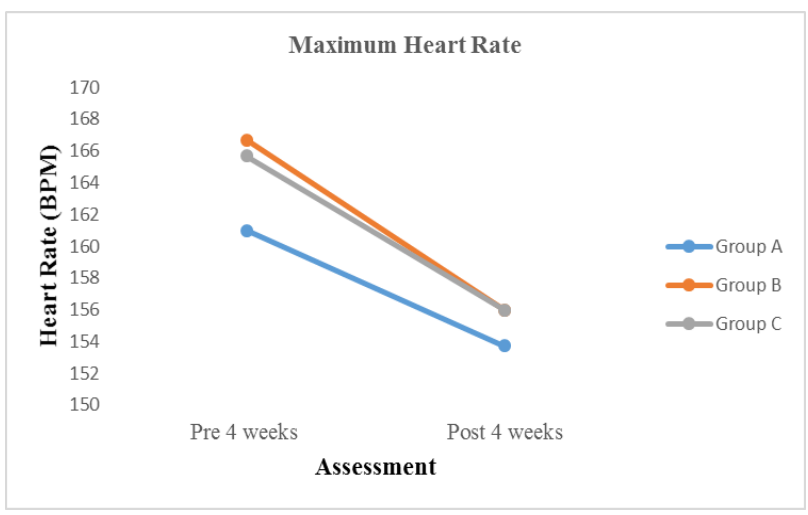

Figure 3 Maximum heart rate before and after four weeks of intervention (BPM = beat per minute), *Pre-post comparison, $\mathrm{P}$ $\leq 0.05$

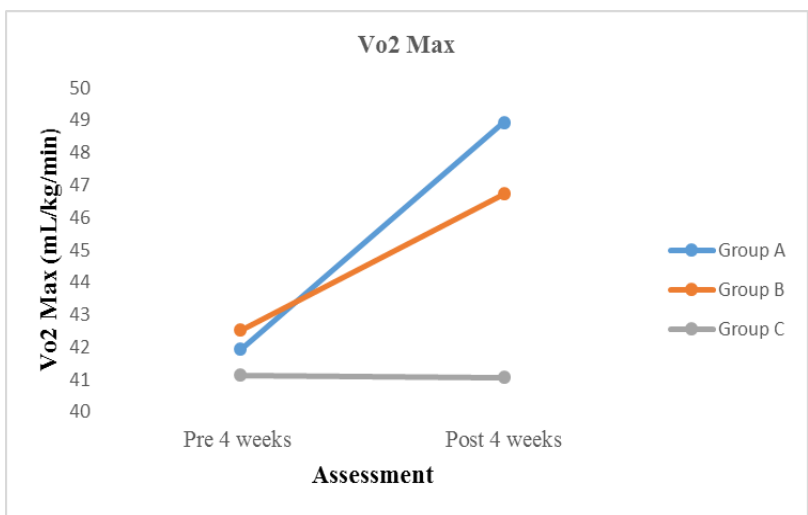

Figure $4 \mathrm{VO}_{2}$ max before and after four weeks of intervention, *Pre-post comparison, $\mathrm{P} \leq 0.05$

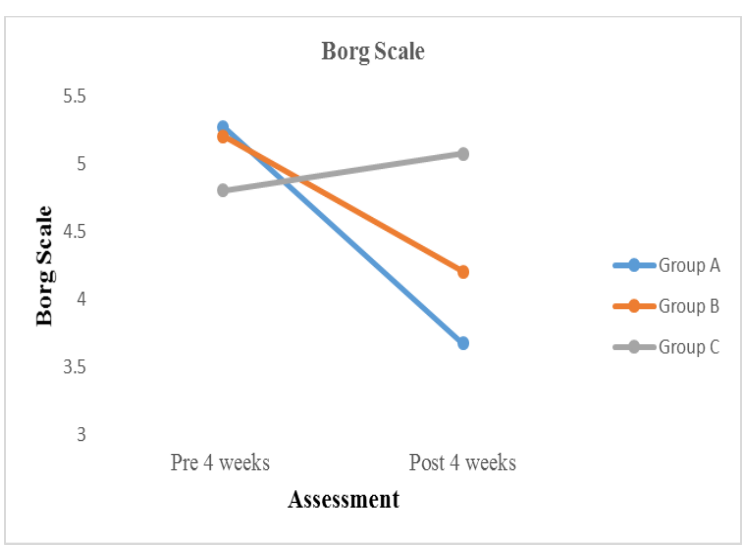

Figure 5 Rating of perceived exertion before and after four weeks of intervention, *Pre-post comparison, $\mathrm{P} \leq 0.05$

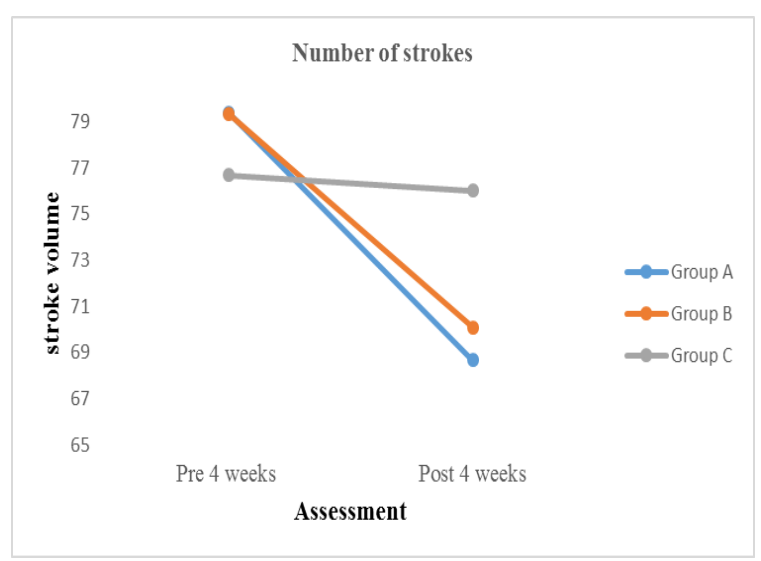

Figure 6 Number of strokes before and after four weeks of intervention, *Pre-post comparison, $\mathrm{P} \leq 0.05$

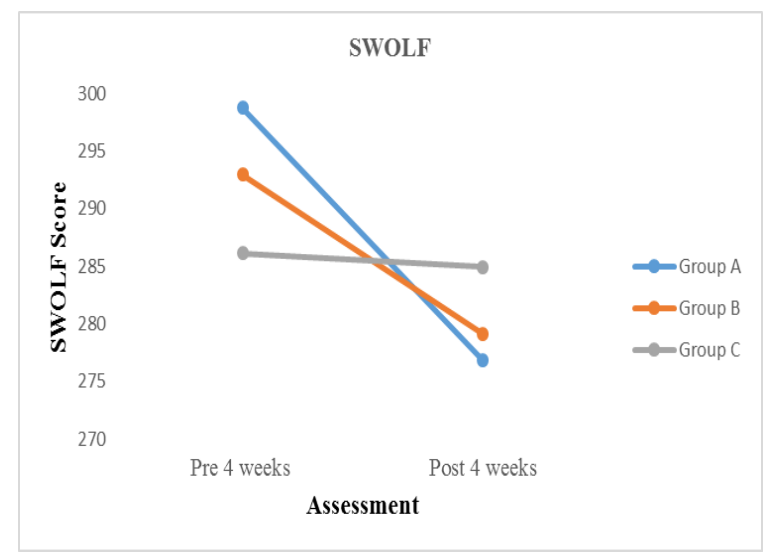

Figure 7 SWOLF Score before and after four weeks of intervention, *Pre-post comparison, $\mathrm{P} \leq 0.05$

Journal of Experimental Biology and Agricultural Sciences http://www.jebas.org 
For all the three groups, participants were scheduled with training sessions of five days weekly and an adequate amount of rest was given between various parts of training sessions. The training sessions and progression was monitored by an expert swimming coach whereas the RMT training and levels were prescribed by an expert cardiorespiratory physiotherapist. The study procedure is explained in Figure 1.

\subsection{Instruments}

The measurement of SWOLF, swim distance, heart rate, and Vo2max were recorded using the Garmin Forerunner 935 watch. The Garmin was connected to the smartphone through the Garmin app to record the data. To measure the heart rate accurately, the Garmin watch was strapped on the chest with a sensor connecting to the watch and it was worn throughout the $100 \mathrm{~m}$ swimming distance. Precautions were taken to prevent the strap from restricting the breathing of the participant or creating any discomfort during the swimming.

Respiratory muscle training was given with the 'Power breathe ${ }^{\circledR}$ ' medium resistance trainer (Nepomuceno et al., 2016). This medium resistance device was chosen for intermediate users. All the participants using the power breath were given clear guidance with a practical demonstration before given to them. This trainer has different levels of resistance setting starting from level 0 to level 9 (Amaro et al., 2017). An adjustable knob at the bottom of the device helps to increase or decrease the resistance as the higher level, the more is resistance. For the current study, level 2 to 8 has been prescribed for different groups with different exercise sessions.

\subsection{Statistical Analysis}

Shapiro-Wilk test was used to check the distribution of the data \& all the data were found to be normally distributed. Mean \pm standard deviation was used to present the values of each variable. For statistical analysis, the differences within the group were analyzed using paired T-test while the difference between the groups was assessed using repeated measure two-way ANOVA. The significant level was set at 0.05 with $95 \%$ confidence level analyse.

\section{Results}

\subsection{Demographics details of the subjects}

In the current study, forty-five subjects were recruited. The baseline demographics details of the subjects were presented according to the group allocation in Table 1 .

3.2 Effects of respiratory muscle training (RMT) on swimming performance

Journal of Experimental Biology and Agricultural Sciences

http://www.jebas.org
The four weeks of respiratory muscle training among fifteen collegiate swimmers have shown significant improvement in the swimming performance $(p<0.05)$ except the time taken for the swimmers to complete the $100 \mathrm{~m}$. The results are presented in Table 2.

3.3 Effects of combined respiratory muscle training and individualized training (RMT + IT) on swimming performance

The differences of combined respiratory muscle and individualized training were evaluated pre and post 4 weeks intervention among fifteen swimmers. All the variables showed significant improvement except the time taken for the swimmers to complete the $100 \mathrm{~m}$ distance similar to the respiratory training muscle group (Table 3).

3.4 Comparison between intervention (RMT+IT, RMT, and TS) on swimming performance

A repeated measure two-way ANOVA was used to compare the three groups (Two interventions and one control). A significant differences were found between the groups $\{F(17,238)=1483.02$, $\mathrm{p}<0.05, \mathrm{np} 2=0.991\}$ and the improvement within the group after four weeks of intervention $(\mathrm{F}(1,14)=35.88, \mathrm{p}<0.05$, np2 $=0.719)$.

Besides, the interaction of the group $\times$ time also showed significant effect $(\mathrm{F}(17,238)=8.385, \mathrm{p}<0.05, \mathrm{np} 2=0.375)$. All the results showed a large effect size as there is a clear significant relationship between the group and the improvement over 4 weeks. Figure 2 - 7 present the swimming performance in comparison of the three groups.

\section{Discussion}

The present study showed that both intervention groups; RMT+IT and RMT showed significant improvement in swimming performance after four weeks of intervention except for the time taken to complete the $100 \mathrm{~m}$ distance. The comparison between the three groups reported significant differences as the combined intervention of RMT+IT showed better improvement in the time taken to complete the $100 \mathrm{~m}$ distance, max HR, Vo2 max, perceived exertion, number of strokes, and the SWOLF score compared to isolated RMT and TS.

In this study, isolated RMT has shown improvement in the swimming performance among 15 collegiate swimmers after four weeks as there is a significant difference in max HR, Vo2 max, Borg scale, number of strokes as well as the SWOLF score. This is similar to previous studies as meta-analysis on the impact of respiratory muscle training on the performance among various athletes has reported excellent improvement in terms of athletic performance, respiratory muscle strength, and endurance in 
comparison to usual training (HajGhanbari et al., 2013). The same meta-analysis has also stated that there is an improvement in aerobic metabolism and oxygen delivery due to changes in aerobic capacity of primary and accessory respiration muscle after RMT intervention (HajGhanbari et al., 2013). Indirectly, these changes have contributed to the delayed onset of fatigue and decrease competitive blood flow (Harms, 2007; Witt et al., 2007). Although the previous study on acute and chronic pulmonary responses has reported that with an increase of respiratory muscle strength, the pulmonary functions also improved concurrently (Yilmaz \& Özdal 2019). Interestingly, this study also has reported the correlation between the detriments of respiratory muscle fatigue with an increase in respiratory muscle strength (Yilmaz \& Özdal 2019). Thus, it can be concluded that with regular RMT, the swimming performance can be enhanced further.

Despite the improvement shown in the RMT group, the current study intended to observe the changes in swimming performance by incorporating both respiratory muscle training and individualized training among the collegiate swimmers. The results have shown a vast difference in the swimming performance comparing to isolated respiratory muscle training or the usual training performed by the collegiate swimmers. The combined intervention of both RMT and IT has shown tremendous improvement in the time taken to complete the $100 \mathrm{~m}$ distance, max $\mathrm{HR}, \mathrm{VO}_{2}$ max, perceived exertion, number of strokes, and the SWOLF score. Individualized training has been used to improve the performance among various sports including marathon and distance runners; football, soccer, tennis, rugby, and others (Manzi et al., 2009; Manzi et al., 2012; Ulbricht et al., 2013; Jiménezreyes et al., 2017; Cavaggioni et al., 2019).

In a study conducted among youth elite football players, IT has been regarded as an effective approach in enhancing the player's performance (Mujika et al., 2007). Similarly, in another study among distance runners, IT has shown an increased endurance level after eight weeks of training (Manzi et al., 2009).

The ballistic (jumping) performance has also shown significant improvement with the IT approach compared to the normal resistance training among forty-eight trained soccer and rugby players (Jiménez-reyes et al., 2017).

Although IT has been used to increase performance among various sports, to the best of our knowledge, there is no study has been conducted among swimmers using the IT approach.

The novelty of the current study to combine both the intervention of RMT and IT among the swimmers to improve various parameters (HR, Stroke Volume, $\mathrm{VO}_{2}$ max, perceived exertion, and SWOLF score), which may further increase the swimmer's performance especially among the athletic swimmers.
The main limitation of the current study is the small sample size of swimmers in each intervention group. Despite this, the researchers in the current study have followed stringent \& consistent observations leading to significant results statistically.

Further, the study has been conducted only for a short duration of time. Future studies were encouraged to be conducted in a longer duration of time to assess the chronic adaptations of RMT and IT on swimming performance with larger sample size, especially among the athletic swimmers

\section{Conclusion}

The results of the current study indicate that the combined intervention of RMT and IT has shown better improvement compared to the isolated RMT and TS among the swimmers. The result suggests that training coaches can adapt the RMT and IT approach in their training to cater to the specific needs of the swimmers to enhance the development and performance, especially among athletic swimmers.

\section{Acknowledgements}

The authors would like to thank INTI International University for the work supported by INTI IU Research Seed Grant 2018: INTIFHLS-01-05-2018.The authors would like to thank INTI International University for the work supported by INTI IU Research Seed Grant 2018: INTI-FHLS-01-05-2018.

\section{References}

Amaro NM, Marinho DA, Marques MC, Batalha NP, Morouço PG (2017) Effects of Dry-Land Strength and Conditioning Programs in Age Group Swimmers. The Journal of Strength \& Conditioning $\begin{array}{llll}\text { Research } & 31(9) \text { : } & \text { 2447-2454. }\end{array}$ 10.1519/JSC.0000000000001709.

Cavaggioni L, Trecroci A, Tosin M, Iaia FM (2019) Individualized Dry-Land Intervention Program for an Elite Paralympic Swimmer. Journal of Sports Medicine and Physical Fitness 59(1): 82-86. DOI: 10.23736/S0022-4707.18.08242-7.

Cox KL, Burke V, BeilinLJ, Grove R (2006) Blood Pressure Rise with Swimming versus Walking in Older Women: The Sedentary Women Exercise Adherence Trial 2 (SWEAT 2). Journal of Hypertension 24(2): 307-314.

Cunha M, Mendes F, Paciência I, Rodolfo A, Carneiro-Leão L, Rama T, Rufo J, Delgado L, Moreiraa A (2019) The Effect of Inspiratory Muscle Training on Swimming Performance, Inspiratory Muscle Strength, Lung Function, and Perceived 
Breathlessness in Elite Swimmers. Porto Biomedical Journal 4(6): e49. doi: 10.1097/j.pbj.0000000000000049.

GarridoN, Marinho DA, Reis VM, Roland van den Tillaar R, Costa AM, Silva AJ, Marques MC (2010) Does Combined Dry Land Strength and Aerobic Training Inhibit Performance of Young Competitive Swimmers?" Journal of Sport Science and Medicine 9(2): 300-310.

Grant MC, Kavaliauskas M (2017) Land Based Resistance Training and Youth Swimming Performance. International Journal of Sports and Exercise Medicine 3(4): 1-11.

HajGhanbari B, Yamabayashi C, Buna TR, Coelho JD, et al. (2013) Effects of Respiratory Muscle Training on Performance in Athletes: A Systematic Review with Meta-Analyses. Strength And Conditioning 27(6): 1643-1663.

Harms CA (2007) Insights into the Role of the Respiratory Muscle Metaboreflex. Journal of Physiology 584(3): 711. doi: 10.1113/jphysiol.2007.145540.

Illi SK, Held U, Frank I, Spengler CM (2012) Effect of respiratory muscle training on exercise performance in healthy individuals: a systematic review and meta-analysis. Sports Medicine 42(8): $707-$ 724. DOI: $10.1007 / \mathrm{BF} 03262290$

Jiménez-reyes P, Samozino P, Brughelli M, Morin J (2017) Effectiveness of an Individualized Training Based on Force Velocity Profilingduring Jumping.Frontiers in Physiologydoi: 10.3389/fphys.2016.00677.

Lavin KM, Guenette JA, Smoliga JM, Zavorsky GS (2015) Controlled-Frequency Breath Swimming Improves Swimming Performance and Running Economy. Scandinavian Journal of Medicine and Science in Sports 25(1): 16-24.

Lemaitre F, Coquart JB, Chavallard F, Castres I, Mucci P, Costalat G, Chollet D (2013) Effect of Additional Respiratory Muscle Endurance Training in Young Well-Trained Swimmers. Journal of sports science \& medicine 12(4): 630-638.

ManziV, Iellamo F, Impellizzeri F, D'Ottavio S, Castagna C (2009) Relation between Individualized Training Impulses and Performance in Distance Runners. Medicine and Science in Sports and Exercise 41(11): 2090-2096. DOI: 10.1249/MSS.0b013e3181a6a959.

Manzi V, Castagna C, Padua E, Lombardo M, D'Ottavio S, Massaro M, Volterrani M, Iellamo F (2012) Dose-Response
Relationship of Autonomic Nervous System Responses to Individualized Training Impulse in Marathon Runners. American Journal of Physiology-Heart and Circulatory Physiology 296(6):

H1733-1740.

Mujika I, Santisteban JM, Angulo P, Padilla S (2007) Individualized Aerobic-Power Training in an Underperforming Youth Elite association football Player. International Journal of Sports Physiology and Performance 2(3):332-335.

Nepomuceno J, Ventura BR, Gómez TB, Neto MG (2016) Use of Powerbreathe ${ }^{\circledR}$ in Inspiratory Muscle Training for Athletes: Systematic Review. Fisioterapiaem Movimento 29(4): 821-830.

Sadowski J, Mastalerz A, Gromisz W, Niźnikowski T (2012) Effectiveness of the Power Dry-Land Training Programmes in Youth Swimmers. Journal of Human Kinetics 32(1): 77-86.

Szczepan S, Danek N, Michalik K, Wróblewska Z, Zatoń K (2020) Influence of a Six-Week Swimming Training with Added Respiratory Dead Space on Respiratory Muscle Strength and Pulmonary Function in Recreational Swimmers. International Journal of Environmental Research and Public Health 17(16): 5743. Doi: 10.3390/ijerph17165743.

Tovin BJ (2006) Prevention and Treatment of Swimmer's Shoulder. North American journal of Sports Physical Therapy 1(4): 166-175.

Ulbricht A, Fernandez-fernandez J, Ferrauti A (2013) Conception for Fitness Testing and Individualized Training Programs in the German Tennis Federation. Sport-Orthopaedie - SportTraumatologie 29(3): 180-92. http://dx.doi.org/10.1016/j.orthtr.2013.07.005.

Witt JD, Guenette JA, Rupert JL, McKenzie DC, William Sheel A (2007) Inspiratory Muscle Training Attenuates the Human Respiratory Muscle Metaboreflex. Journal of Physiology 584(3): 1019-1028. doi: 10.1113/jphysiol.2007.140855.

Yasemin B, Dağlıŏlu O, Bostanc1 O (2019) The Effect of Respiratory Muscle Training on Aerobic Power and Respiratory Parameters in Swimmers. UluslararasiSporEgzersizveAntrenman Bilimi Dergisi 5(4): 214-20.

Yilmaz ÖF, Özdal M (2019) Acute, Chronic, and Combined Pulmonary Responses to Swimming in Competitive Swimmers. Respiratory Physiology and Neurobiology 259: 129-135. https://doi.org/10.1016/j.resp.2018.09.002. 